\title{
Arsenal of microfluidic testing devices may combat COVID-19 pandemic
}

\author{
By José Alvim Berkenbrock, Rafaela Grecco-Machado, and Sven Achenbach
}

\section{COVID-19}

The SARS-CoV-2 coronavirus that causes the disease COVID-19 is responsible for the most severe worldwide virus outbreak in recent history. The first cases of COVID-19 were registered in China in December 2019, and it has since rapidly spread around the globe. Many scientists and physicians have been working to identify this new virus and to fight the infection. The high number of cases around the world led the World Health Organization (WHO) to declare a pandemic situation on March 11, 2020. Although viruses have a variety of arrangements and compositions, they are generally composed of genetic material (DNA or RNA), a protein shell, and a lipid bilayer. Detection approaches focus either on the proteins that form the shell or on the genetic material and its sequence of nucleotides, the building blocks of DNA and RNA.

The first publications on SARSCoV-2 identified it as an RNA-based virus of the order Nidovirales, the family Coronaviridae, and the genus Betacoronavirus. Although many coronavirus infections in humans cause only mild symptoms, SARS-CoV-2 was determined to be similar to other aggressive strains, such as the Severe Acute Respiratory Syndrome coronavirus (SARS-CoV) and

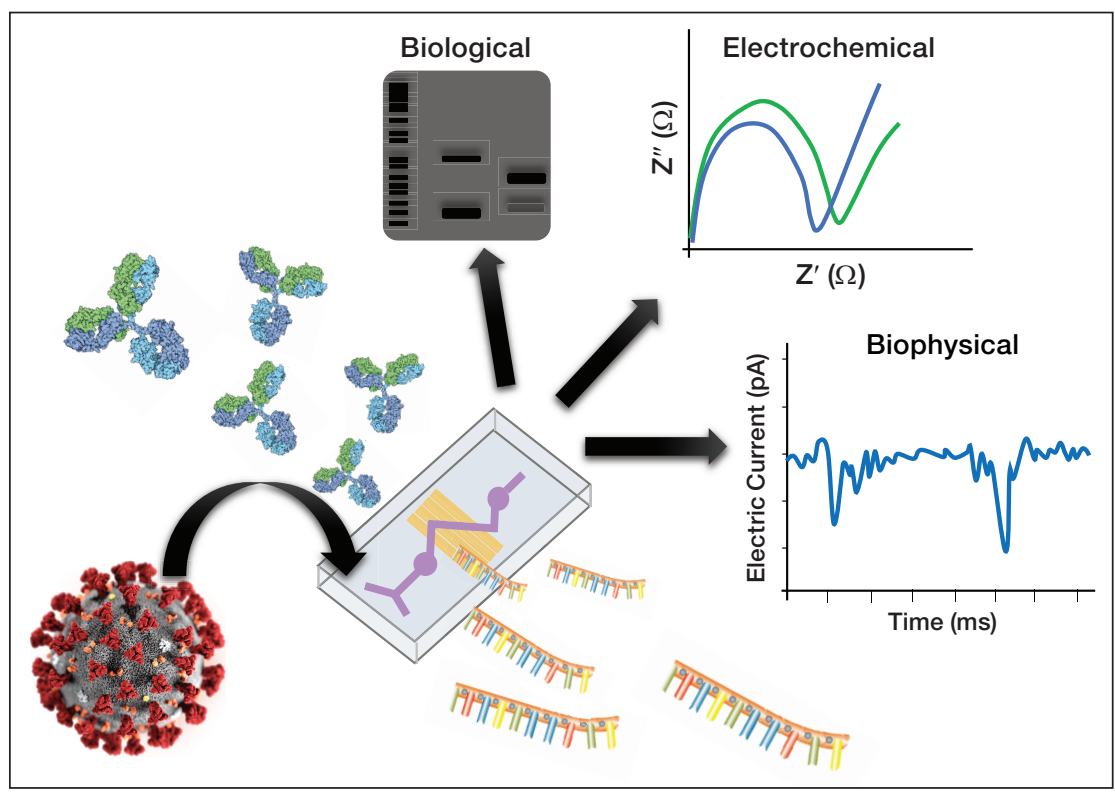

Figure 1. The use of microfluidic devices for the detection and manipulation of viruses. SARSCoV-2, represented on the lower-left corner, can potentially be detected by microfluidic devices. Antibodies and primers are elements commonly used for the detection of target elements in samples. Microfluidic devices rely on well-established methods for analyzing samples, including genetic amplification (bands in an electrophoretic gel), analysis of impedance, and detection by translocation events is based on pulses in the electric current due to partial and non-permanent obstruction of nanopores. Credit: José Alvim Berkenbrock. the Middle East Respiratory Syndrome coronavirus (MERS-CoV). The similarities among SARS-CoV-2, SARS-CoV, and MERS-CoV provided scientists with a strong indication of where to start working. Even before samples from patients infected with SARS-CoV-2 became available, Christian Drosten, director at the Institute of Virology at Berlin's Charité University Hospital, together with an international research group, published a WHO-endorsed diagnostic protocol for SARS-CoV-2 based on its similarities to other viruses. ${ }^{1}$ Currently, new information about SARS$\mathrm{CoV}-2$ continues to be published daily. To date, the most effective measures to contain the spread of SARS-CoV-2 have been social distancing, testing widely, isolating and closely monitoring infected patients, and tracing the people who had contact with them (See Figures 1 and 2).

\section{Benchtop assays slow to test for COVID-19}

Testing is key to identifying infected individuals so they can be isolated and treated and so that deaths related to the limited capacity of health systems can be avoided. A news feature published in Nature observed how the United Arab Emirates, Singapore, and South Korea required their industries to produce sufficient screening test kits and required extensive testing of the population as well as the tracing of each patient's circle of contacts. ${ }^{2}$ A variety of experiments are being developed to test for COVID-19, but the technique known as real-time reverse transcriptionpolymerase chain reaction (qRT-PCR) is the most widely used. Recommended by both the WHO and the US Centers for Disease Control and Prevention (CDC), this test uses a biological sample extracted 
from the patient's nasopharyngeal swabs. This sample is tested for the presence of viral genetic material. Some of the key elements necessary for qRT-PCR are thermocyclers to amplify the complementary DNA, primers to bind to the target sequence, and buffers that enable enzymes to function correctly. These elements allow for the amplification of the viral genetic material in the patient's samples to obtain a measurable result.

Despite the ability of the qRT-PCR test to detect viral material in patient samples, it has substantial drawbacks. First, the test is a lengthy process. Although in the best cases reported, the time to collect samples, perform the reaction, and analyze the results takes from 60 minutes to 4 hours, this process typically takes a few days to complete. Other time-limiting factors include extracting the sample from the patient, storing and delivering the sample to the testing center, and reporting results to the physicians who requested the test (not always an online process).

Second, shortages of biotechnology products such as enzymes, buffers, and primers can, as has occurred during the current pandemic, result in a bottleneck for widespread testing of populations. Third, only a few companies have the capacity to produce the qRT-PCR test kits.

The rapid spread of the virus has obliged governments to import large amounts of the test kits. While larger economies can import tests, many countries are facing shortages and have not been able to perform enough tests in their populations. These limitations on the production of screen tests have reduced the chances of punctual isolation, jeopardized positive outcomes for thousands of patients, and have become a potential threat to the safety of thousands of people worldwide.

\section{Microfluidic devices offer alternative to benchtop assays}

Alternatives to the problematic benchtop assays such as real-time RT-PCR can be found in the field of micro- and nanofabrication. Advancements achieved by the electronics industry have allowed for the fabrication of microdevices for fine manipulation of fluids. Microfluidics is

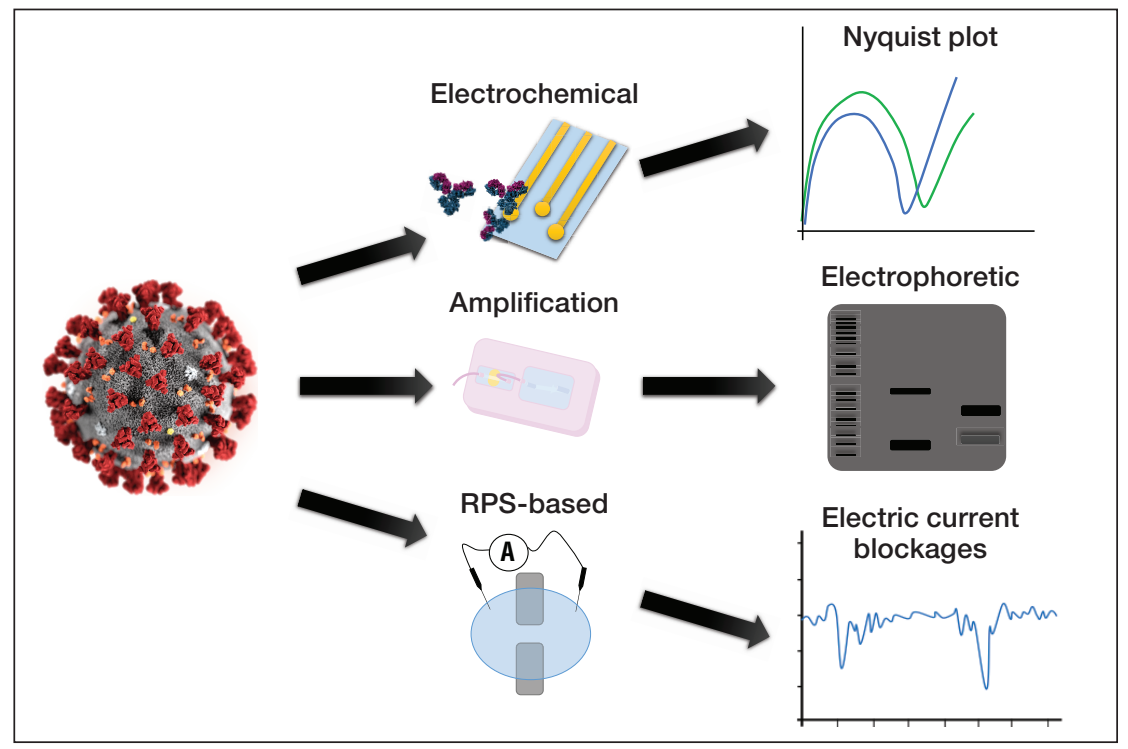

Figure 2. The use of microfluidic devices for the detection and manipulation of viruses. SARSCoV-2, represented on the left, can potentially be detected by different approaches with microfluidic devices. Detection approaches include the modification of electrode surfaces inducing changes in the measurements of impedance, the amplification of the viral genetic material in order to generate a sufficiently large amount of copies to enable visualization, and translocation events through nanopore-based devices known as resistive pulse sensors (RPS). Credit: José Alvim Berkenbrock.

a field of applied sciences that pairs the study of fluids and fabrication of devices with at least one dimension in the micrometer range. Similarly, nanofluidics deals with devices with dimensions in the nanometer range.

In the last few decades, this field has rapidly evolved to create new solutions for health-related applications. The presence of microfluidics in the health sciences has become evident in lab-ona-chip, organs-on-a-chip, and point-ofcare testing (PoCT) devices. Sometimes referred to as "bedside testing," PoCT devices facilitate the extraction of information from patient samples without extensive laboratory work. Although these devices do not always achieve the same levels of sensitivity and reliability as the well-established laboratory assays, they offer a number of options for screen testing. Microfluidics-based testing devices have several advantages over traditional methods: the ability to extract information from reduced sample volumes; decreasing the need for reagents, waste, and sometimes energy consumption; shorter reaction times since smaller volumes are processed; and parallel operations for multiple concurrent detections.
Devices rely on fluorescent elements Several studies have demonstrated the versatility of this class of devices. Currently, at least three approaches exploit fluorescent elements to indicate detection and signal to the user presence of the target element (e.g., a virus). Dependent on the fluorescent elements, called labels, such a group of devices have the basic triad of binder, target, and flag (see Figure 3).

In a recent article published in Biosensors and Bioelectronics, M.-G. Kim and colleagues from the Gwangju Institute of Science and Technology in South Korea used a paper-based device to differentiate three viruses: ZIKV (Zika), CHIKV (chikungunya), and DENV (dengue), all spread by the same vector: the Aedes aegypti mosquito. ${ }^{3}$ Like SARS$\mathrm{CoV}-2$, the viruses used in this study have RNA as their genetic material.

The research team obtained positive results when detecting the viruses' genetic material, both in a phosphate buffered saline (PBS) control and in serum samples obtained from patients. The researchers identified a single copy of ZIKV RNA in PBS and 10 copies in a patient's serum in a relatively quick process (within 1 hour from RNA extraction). In this system, the 
fluorescence of the wells only decreased upon amplification of the target sequence contained in the sample. The wells without the target virus remained shiny while those with samples containing the target became dark. The detection principle is based on reverse transcription loop-mediated isothermal amplification (RT-LAMP), a technique for the amplification of RNA. This technique differs from PCR in requiring more primers to bind the target. Additionally, while PCR needs multiple temperature cycles, LAMP requires only one temperature. Thus, LAMP can use a simpler technology apparatus that allows portable equipment to perform PoCT.

Another example of portability potential is evident in work by Mengqi Kong of Zhejiang University and colleagues from China and Singapore. They developed a wearable device to identify HIV (AIDS) that used the body's temperature to support the amplification reaction. ${ }^{4}$ The copies of viruses in a sample were increased in an isothermal amplification reaction with the heat produced by the human wrist $\left(33-34^{\circ} \mathrm{C}\right)$. Since the patient samples may contain only a few copies of the virus, the amplification reaction expands the number of copies and facilitates detection and signaling. This polymer-based device detected a concentration of 100 copies of HIV-1 per $\mathrm{mL}$ of solution in a 24-minute-long assay. The research team developed a smartphone-based scanner for fluorescence results.

Another 2019 study, by Carlton F.O. Hoy of The University of Tokyo and colleagues from Japan, focused on detecting MERS-CoV using a fluorescently linked immunosorbent assay. ${ }^{5}$ The research team used a polymer cassette to perform a MERS immunoassay and used immobilized His-MERS-NP (protein) to detect anti-MERS-NP (antibodies) in samples. Although experiments with patient samples are still to be done, the initial laboratory results showed a fair limit of detection of $200 \mu \mathrm{g} / \mathrm{mL}$.

\section{Devices use alternative signaling methods}

Label-free detectors are a class of devices that avoid extra reagents for assays by using signaling methods other than fluorescence. H.A.M. Faria and V. Zucolotto of the Universidade de São Paulo in Brazil presented an electrochemical DNA biosensor for ZIKV. ${ }^{6}$ Their sensor is based on gold electrodes functionalized with thiol chemical groups. Thiol functional groups form a self-assembling monolayer on gold surfaces that facilitate the attachment of antibodies (or DNA probes) to the device (see Figure 4). The research group used impedance analysis to observe the variations caused by adding chemical layers (thiol), binding elements (DNA probe), and finally to recognize the virus detection by the electrodes. Similarly, P.R. Bueno and colleagues at São Paulo State University presented a device to detect DENV based on the electrochemical capacitive method. ${ }^{7}$ They proposed a new label-free and reagentless methodology based on a ferrocene-tagged peptide

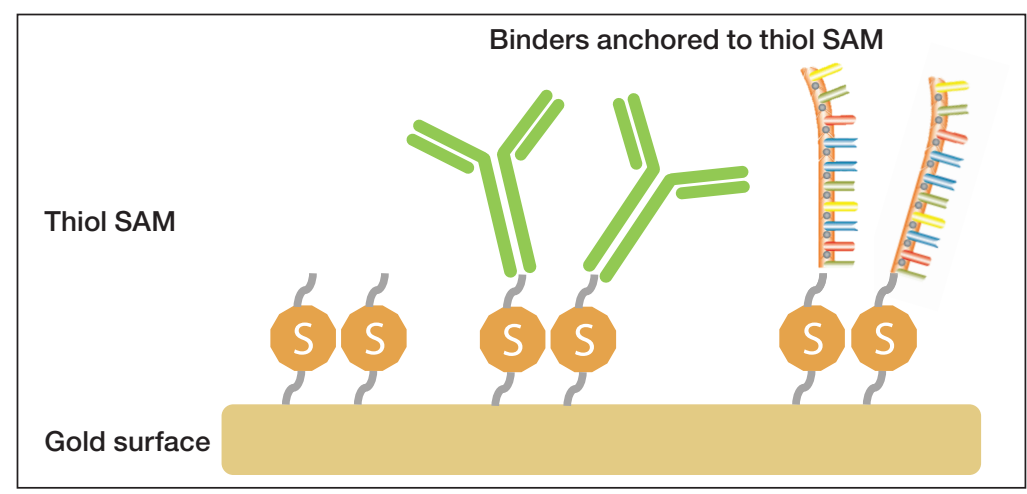

Figure 4. Scheme of a thiol self-assembling monolayer (SAM) on gold with an antibody or primers anchored. Different elements can be anchored on thiol SAM although it might need other chemical ligands to enable the binding. Credit: José Alvim Berkenbrock.

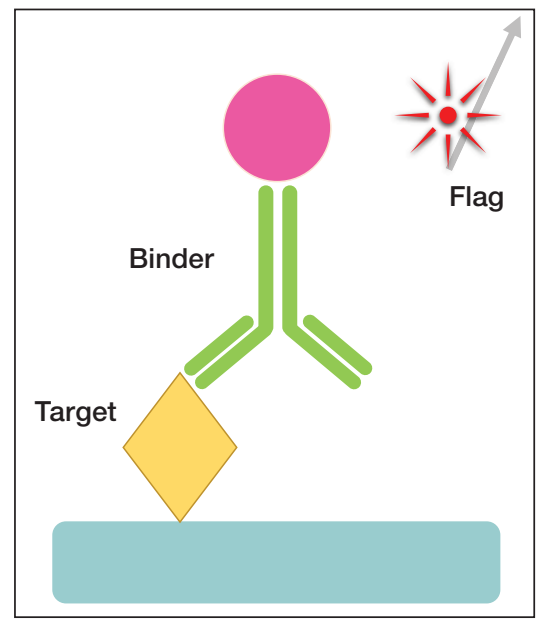

Figure 3. Example of basic elements in a labelbased detector. The target (yellow lozenge) is recognized by the binding element (an antibody) that contains the signaling particle. Upon binding, the flag produces a colorimetric indication of detection. Credit: José Alvim Berkenbrock.

attached to antibodies. The proposed detection principle was based on altering the surface capacitance of the electrode upon antigen-antibody binding.

The two research groups were able to identify a low concentration of target elements without using labels or amplification steps. However, more complex electronics were necessary to process the electrical measurements. Both teams used electrochemical impedance spectroscopy to analyze the samples on their electrode surfaces, which were modified using two different approaches. To bind the target to the electrodes, Faria and Zucolotto used a primer for a specific region of the target's DNA (i.e., NS5), while Buenos's team applied an antibody to capture a specific protein (i.e., NS1) in the sample. For SARS$\mathrm{CoV}-2$, specific regions of its genetic code already used for qRT-PCR may be adapted for such microfluidics devices. The labelfree detectors can reduce the number of reagents and be extended to platforms for the detection of multiple targets.

\section{Emerging technologies yield innovative microfluidic devices}

Besides effective microfluidic detectors that rely on well-established techniques such as electrochemical measurements and immunoassays, emerging technologies and innovative micro- and 
nanofabrication capabilities have been used for novel devices. Research published this year by Gwo-Bin Lee and colleagues from National Tsing Hua University, Taiwan, in the journal Lab on a Chip reported on aptamers attached to magnetic beads to detect H1N1 (Influenza A). ${ }^{8}$ Aptamers are single-stranded oligonucleotides that bind to very specific genetic targets. After the aptamer-coated beads captured the viruses, two types of antibodies were bound to the viruses for signaling purposes. Moreover, the research team proposed using an electromagnetic field for manipulation and mixing individual droplets on a superhydrophobic table without the need for channels to carry the fluids.

Hsueh-Chia Chang of the University of Notre Dame and colleagues proposed another variation from classical approaches in research published this February in Sensors and Actuators B: Chemical. ${ }^{9}$ The research team developed a porous membrane functionalized with specific short stretches of single-stranded RNA called oligoprobes to detect and discriminate four subtypes of DENV (DENV-1 to 4). The team was able to detect down to 100 copies of RNA per $\mathrm{mL}$ in human plasma in about 90 minutes. In the proposed platform, a single PCR was performed to interrogate the sample against the four subtypes of the virus. Then the product was filtered, and each of the subtypes four specific reservoirs electrically revealed the presence of the target subtype. An ion-selective membrane was attached to each of the reservoirs to selectively detect the virus subtypes by a system monitoring the ion current through the membranes.

The fabrication of membranes with nanopores for microfluidic detectors may require sophisticated technological equipment such as focused ion beams and cleanroom facilities. Vincent Tabard-Cossa and colleagues from the University of Ottawa proposed an ingenious new technique for the fabrication of nanopores in silicon nitride membranes $\left(\mathrm{SiN}_{x}\right)$, which was described in the journal Nature Protocols. ${ }^{10}$
By applying an electric field to $\mathrm{KCl}$ solution around $\mathrm{SiN}_{x}$ membrane, the research team opened pores with diameters of 1-20 $\mathrm{nm}$. Their work has shown promising results in detecting generic double- and single-stranded DNA. The team suggested that this fabrication method could democratize access to nanopore-based membranes for researchers globally.

Researchers from Imperial College London presented another way of making the fabrication of microdetectors more accessible in a non-peer-reviewed paper recently made available at bioRxiv. ${ }^{11}$ Corresponding authors Estefania NunezBajo and Firat Güder, with colleagues also from the University of Turku (Finland) and Moredun Research Institute (Scotland), proposed a silicon-based microdevice to chemically amplify and detect pathogenspecific nucleic acid sequences. Initial experiments were performed with synthetic SARS-CoV-2 since patient samples were not available. Also, the manuscript detailed the fabrication of the chip and electronics setup to enable easy replication of the device and use for screening. These last two works demonstrate simple fabrication processes that can facilitate the widespread production of PoCT devices.

\section{Conclusion}

Although it may take some time for microfluidic detectors for the new SARS-CoV-2 to become widely available, the experience of researchers in this field will be critical. As Drosten and colleagues were able to establish a detection protocol even before they had viral samples, it is expected that engineers will be able to use the knowledge as summarized to create SARS-CoV-2specific microdetectors.

The similarities among viruses and the flexibility of the approaches presented here need to be harnessed to generate rapid tests that are more widely available, easier to use and transport, cheaper to produce, and quicker to generate results than current tests. Critical situations, such as the current pandemic, tend to propel the development of technologies for innovative approaches to detect viruses and explorations of the capabilities of micro- and nanotechnology. New small devices designed to rapidly collect information from a drop of blood or saliva are being developed to detect a variety of diseases and biomarkers. These innovations will accelerate diagnosis of disease, so crucial in a pandemic.

\section{References}

1. V.M. Corman, O. Landt, M. Kaiser, R. Molenkamp A. Meijer, D.K.W. Chu, T. Bleicker, S. Brünink, J. Schneider, M.L. Schmidt, D.G.J.C. Mulders, B.L. Haagmans, B. van der Veer, S. van den Brink, L. Wijsman, G. Goderski, J.-L. Romette, J. Ellis, M. Zambon, M. Peiris, H. Goossens, C. Reusken, M.P.G Koopmans, C. Drosten, Euro Surveill. 25, 1 (2020), doi:10.2807/1560-7917.ES.2020.25.3.2000045.

2. N. Subbaraman, Nature (2020), doi:10.1038/ d41586-020-00827-6.

3. B.S. Batule, Y. Seok, M.-G. Kim, Biosens. Bioelectron. 151, 111998 (2020), doi:10.1016/j.bios.2019. 111998.

4. M. Kong, Z. Li, J. Wu, J. Hu, Y. Sheng, D. Wu, Y. Lin, M. Li, X. Wang, S. Wang, Talanta 205, 120155 (2019), doi:10.1016/j.talanta.2019.120155.

5. C.F.O. Hoy, K. Kushiro, Y. Yamaoka, A. Ryo, M. Takai, Sens. Biosensing Res. 26, 100304 (2019), doi:10. 1016/j.sbsr.2019.100304

6. H.A.M. Faria, V. Zucolotto, Biosens. Bioelectron. 131, 149 (2019), doi:10.1016/.jbios.2019.02.018.

7. J. Cecchetto, A. Santos, A. Mondini, E.M. Cilli, P.R. Bueno, Biosens. Bioelectron. 151, 111972 (2020), doi:10.1016/j.bios.2019.111972.

8. P. Lu, Y. Ma, C. Fu, G. Lee, Lab Chip 20, 789 (2020), doi:10.1039/C9LC01126A.

9. Z. Yin, Z. Ramshani, J.J. Waggoner, B.A. Pinsky, S. Senapati, H.-C. Chang, Sensors Actuators B Chem. 310, 127854 (2020), doi:10.1016/j.snb. 2020.127854

10. M. Waugh, K. Briggs, D. Gunn, M. Gibeault, S. King, Q. Ingram, A.M. Jimenez, S. Berryman, D. Lomovtsev, L. Andrzejewski, V. Tabard-Cossa, Nat. Protoc. 15, 122 (2020), doi:10.1038/s41596-019-0255-2.

11. E. Nunez-Bajo, M. Kasimatis, Y. Cotur, T. Asfour, A. Collins, U. Tanriverdi, M. Grell, M. Kaisti, G. Senesi, K. Stevenson, F. Güder, bioRxiv (forthcoming), doi:10.1101/2020.03.23.002931.

We welcome comments and feedback on this article via email to Bulletin@mrs.org. 